\title{
INFLUENCE OF PRINTING SPEED AND RADIATION DOSE ON THE CURING OF UV INKS AND VARNISHES
}

\author{
Bohumil Jašúrek (D), Jan Vališ (D), Michaela Hozmanová \\ University of Pardubice, Faculty of Chemical Technology, \\ Department of Graphic Arts and Photophysics, Pardubice, Czech Republic
}

\begin{abstract}
This work is focused on curing of UV curable printing inks and varnishes and evaluation of the conversion degree after printing with various printing speeds (between 4 and 10 thousands of sheets per hour) and settings of curing units. Five sets of process offset printing inks (SunCure ${ }^{\circledR}$ FLM - Sun Chemical, Suncure ${ }^{\circledR}$ Starluxe - Sun Chemical, UltraCURA ${ }^{\circledR}$ Sens - Flint Group, NewV Pack MGA ${ }^{\circledR}$ Premium Huber Group, Sicura Litho Nutriplast - Siegwerk) and one UV curable varnish (ExCure All Round EXC90006 - Toyo Ink) were tested. Printing inks and varnish were printed on KBA Rapida $1426+L$ and curing of inks/varnish was examined by Fourier transform infrared spectroscopy. The results show the importance of optimization of both the printing speed and the settings of curing units to achieve appropriate curing connected with good adhesion, no surface tackiness and maximum elimination of migrating components from cured inks that is important mainly in food packaging.
\end{abstract}

Key words: UV ink, UV varnish, curing, polymerization, infrared spectroscopy

\section{INTRODUCTION}

Photoinitiated polymerization of multifunctional monomers and oligomers is one of the most efficient methods to produce highly cross-linked polymer networks. It has found a large number of commercial applications (printing inks and varnishes, coatings, adhesives, etc.), because of its unique advantages (rapid curing time, enhanced material properties, elimination of volatile organic compounds, etc.).

To ensure quality printing and trouble-free subsequent processing of the order is necessary to ensure sufficient ink/varnish drying. Insufficient drying of UV curable inks/varnishes may result in blocking, ink set-off, lower ink/varnish adhesion to printed substrate and increased migration of the printing ink/varnish components. Migration of ink components is very important mainly in food packaging and products intended for children. With this are connected regulations that need to be followed. Between most important belongs Regulation (EC) No 1935/2004 on materials and articles intended to come into contact with food, Regulation (EC) No 2023/2006 about Good Manufacturing Practice for the production of food contact articles, Directive 2007/42/EC relating to materials and articles made of regenerated cellulose, Regulation (EU) No 10/2011 on plastic materials and articles intended to come into contact with food and Swiss ordinance on materials and articles in contact with food (SR 817.023.21) (EUPIA, 2018; Grabitz, 2017). In addition to this, some big companies have their own regulations, for example Nestlé (Nestlé Guidance Note on Packaging Inks) (Nestlé, 2016).

Drying of UV curable printing inks and varnishes is influenced by many factors. Between most important belongs composition of inks/varnishes (type and amount of photoinitiators, monomers, oligomers, pigments, additives), type of UV source (mostly medium pressure mercury lamp or UV LED), UV dose connected with printing speed and intensity of irradiation, presence of inhibitors (oxygen for free radical polymerization and basic substances or humidity for cationic polymerization), thickness of printed ink/varnish, printed substrate etc.

To evaluate the drying of UV curable inks, printers often use simple methods that may not provide the correct information about ink drying. Methods such as Tape test, friction of printing with solvent-impregnated cloth, observing of surface tackiness by touch are used. One of the sophisticated methods for evaluation of drying of UV curable inks is infrared spectroscopy. This method gives information on chemical groups containing polar bonds, or bonds whose dipole moment changes during vibration and from changes of infrared spectra (before and after curing) can be evaluated degree of conversion of cured inks (Škola et al, 2016).

This work is focused on curing of five sets of process offset printing inks (UV curable) and one UV varnish and evaluation of the conversion degree after printing with various printing speeds and settings of curing units. For evaluation of conversion degree, the Fourier transform infrared (FTIR) spectroscopy was used. 


\section{MATERIALS AND METHODS}

This work investigates curing of five sets of process UV offset printing inks from various producers and one UV varnish from TOYO Ink. Tested inks and varnish are summarized in Table 1. One-side coated cardboard Serviliner GD2 (250 g/ $\left.\mathrm{m}^{2}\right)$ was used as printed substrate. All inks and varnish were printed on printing machine KBA Rapida $1426+\mathrm{L}$ equipped with 6 printing units and one varnish unit. Maximum speed of this machine is 15000 sheets/hour with maximum format $1020 \times 1420 \mathrm{~mm}$. The printing speed was changed from 4000 to 10000 sheets/hour (in first step for all inks and varnish from 4000 to 7000 sheet/hour and in second step for well-cured inks at 7000 sheets/hour also at higher printing speeds to 10000 sheets/hour. The power of curing units (equipped with medium pressure mercury lamps) was changed from 70 to $100 \%$ (step $10 \%$ ). On Figure 1 is shown printed sheet (Suncure ${ }^{\circledR}$ Starluxe).

Table 1: Tested inks and varnish

\begin{tabular}{|c|c|}
\hline Producer / ink & Type of ink \\
\hline \multirow{4}{*}{ Fling Group / UltraCURA ${ }^{\circledR}$ Sens } & Process Cyan VW17-508S \\
\hline & Process Magenta VW17-308S \\
\hline & Process Yellow VW17-108S \\
\hline & Process Black VW37-908S \\
\hline \multirow{4}{*}{ Huber Group / NewV pack MGA ${ }^{\circledR}$ premium } & Process Cyan 43UG 5000M \\
\hline & Process magenta 42UG 5000M \\
\hline & Process Yellow 41UG 5000M \\
\hline & Process Black 49UG 5000M \\
\hline \multirow{4}{*}{ Siegwerk / Sicura Litho Nutriplast } & Process Cyan 70-111140-3 \\
\hline & Process Magenta 70-801280-2 \\
\hline & Process Yellow 70-300629-6, \\
\hline & Process Black 70-900479-0 \\
\hline \multirow{4}{*}{ Sun Chemical / SunCure ${ }^{\circledR}$ FLM } & Process Cyan FLM25 \\
\hline & Process Magenta FLM27 \\
\hline & Process Yellow FLM26 \\
\hline & Process Black FLM46 \\
\hline \multirow{4}{*}{ Sun Chemical / Suncure ${ }^{\circledR}$ Starluxe } & Process Cyan USL25 \\
\hline & Process MagentaUSL 27 \\
\hline & Process Yellow USL26 \\
\hline & Process Black USL46 \\
\hline Toyo Ink / ExCure all round EXC90006 & Varnish \\
\hline
\end{tabular}

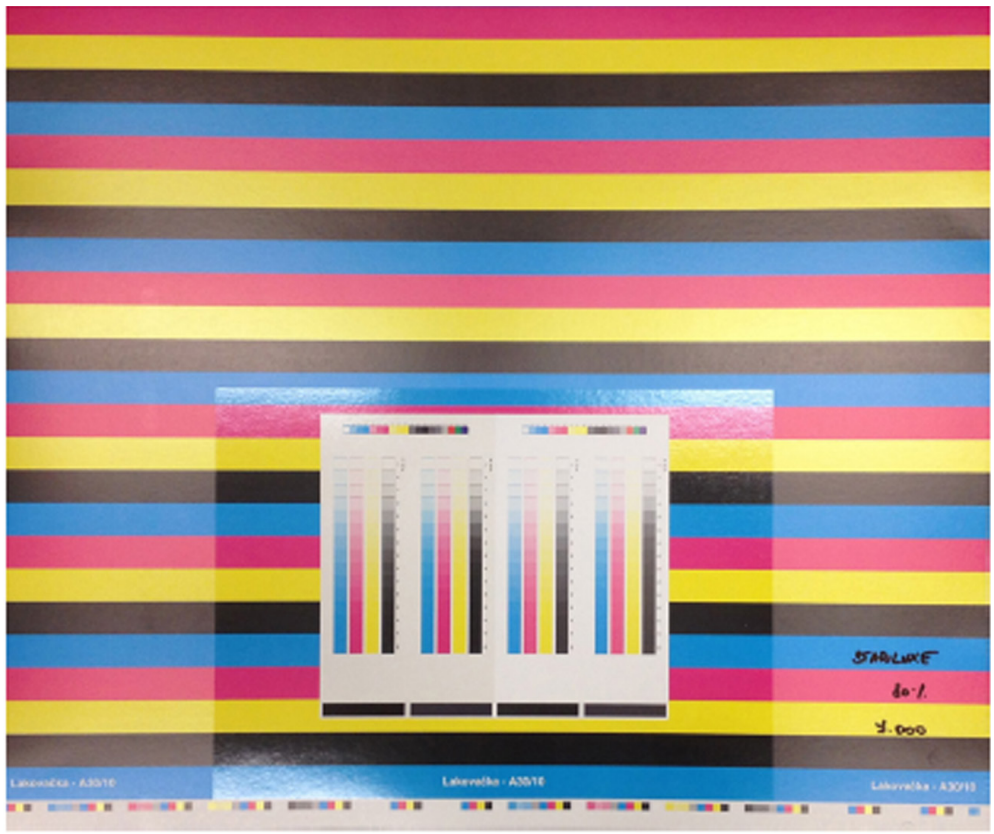

Figure 1: Printed sheet (Sun Chemical/Suncure ${ }^{\circledR}$ Starluxe, 7000 sheets/hour, $80 \%$ ) 
Printed inks (dot area $100 \%$ ) were measured by FTIR spectrometer (Avatar 320, Thermo Scientific, USA) with ATR (Attenuated Total Reflectance) attachment using diamond crystal in range from 4000 to $400 \mathrm{~cm}^{-1}$ at room temperature. Infrared spectrum of every ink at every printing speed was measured four times. Furthermore, the infrared spectrum of uncured inks and varnish was measured. All tested inks and varnish polymerize by free radical polymerization. This type of inks/varnishes is based on acrylate chemistry. The degree of conversion (DC, Equation 1) was evaluated from the decrease of the absorption band (maximum at $809 \mathrm{~cm}^{-1}$ ) area belonging to the acrylate double bond. Carbonyl group (1 $730 \mathrm{~cm}^{-1}$ ) as internal standard was chosen (Colthup et al, 1990).

$D C=1-\left(A_{t} / A_{0}\right) \times 100$

where $A_{0}$ is the ratio of areas of acrylate double bond and internal standard uncured ink/varnish and $A_{t}$ is the ratio of areas of acrylate double bond and internal standard after printing. Figure 2 shows decrease of absorption band of acrylate double bond after curing with two printing speed and different setting of curing unit compared to uncured ink for cyan and magenta (Huber Group, NewV pack MGA ${ }^{\circledR}$ premium). All cyan, yellow and black inks have very similar shape and neighbourhood of absorption band with maximum $809 \mathrm{~cm}^{-1}$. Acrylate double bond of magenta inks (from all producers) and UV varnish partially overlapped with another band. From this reason, area of absorption band $\left(809 \mathrm{~cm}^{-1}\right)$ of magenta inks and UV varnish was evaluated by deconvolution of overlapped bands too.
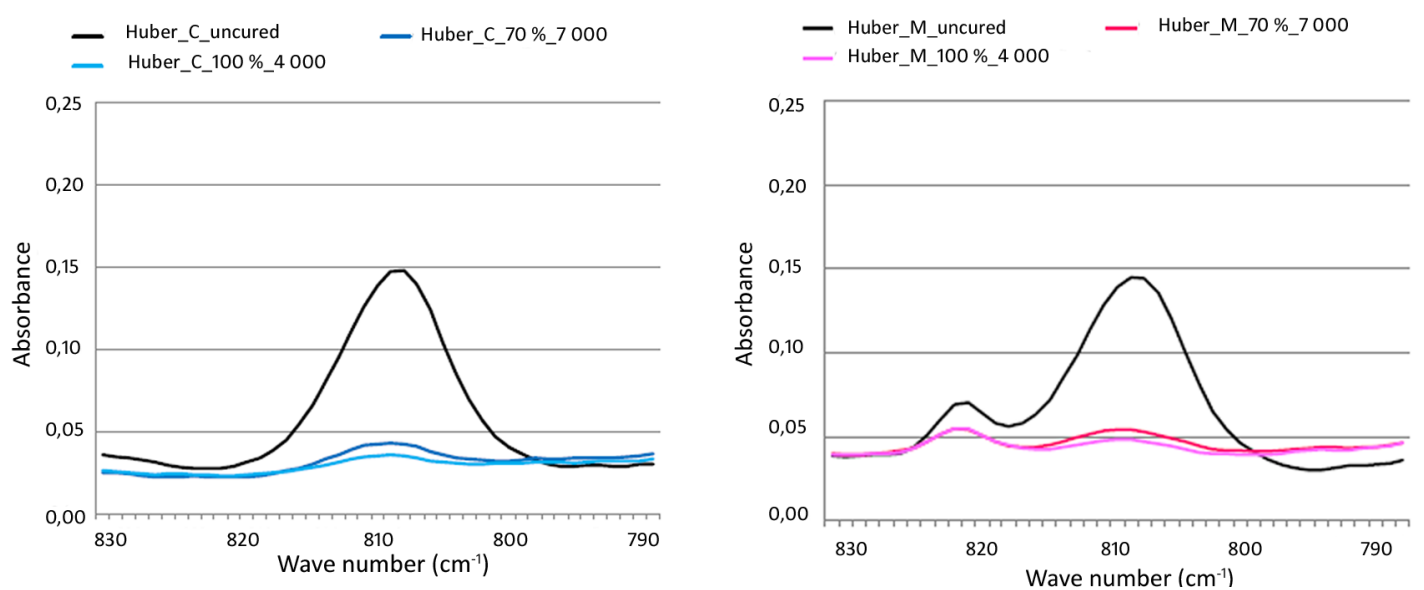

Figure 2: Decrease of acrylate double bond band (max. $809 \mathrm{~cm}^{-1}$ ) during curing (Huber Group, NewV pack MGA ${ }^{\circledR}$ premium). Printing speed 4000 and 7000 sheets/hour, setting of curing unit 70 and $100 \%$. Cyan - left figure, Magenta-right figure.

\section{RESULTS AND DISCUSSION}

Evaluated DC of UV varnish is shown in Figure 3. It is apparent that differences between two ways of evaluation (with and without deconvolution) are minimal (mostly in range of standard deviation).

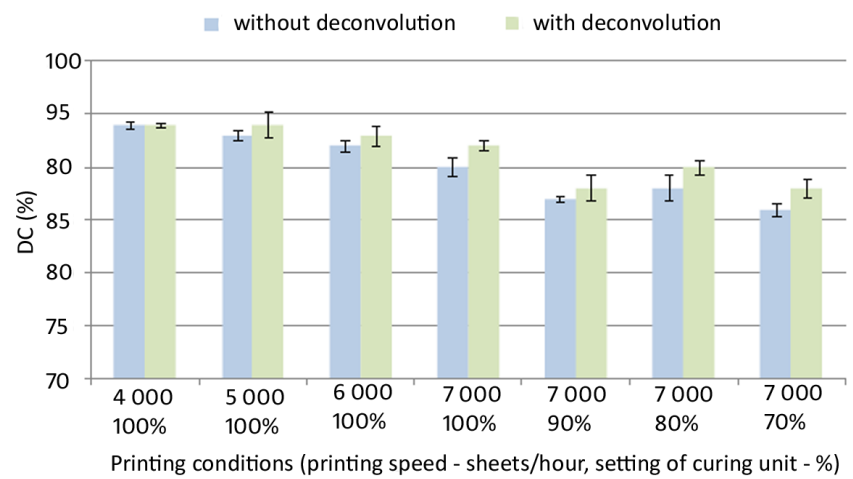

Figure 3: DC of UV varnish (EXCure all round EXC90006 from TOYO Ink) with different printing conditions 
For printing speeds between 4000 and 6000 sheets/hour (curing unit set on 100\%) were reached similar DC and UV varnish was well cured. DC reached with printing speed 7000 and different setting of curing unit were lower and varnish is not fully cured as with lower printing speeds.

Figures 4-8 shown DC of all tested inks. Inks from Flint Group (UltraCURA ${ }^{\circledR}$ Sens) are on Figure 4. It is apparent that all process inks (UltraCURA ${ }^{\circledR}$ Sens) are well cured at speeds 4000 and 5000 sheets/hour. Cyan, yellow and black inks are also well cured at 6000 and 7000 sheets/hour but not magenta inks, where DC decrease and magenta ink is not sufficiently cured at this speeds.

For inks from Huber Group (NewV pack MGA ${ }^{\circledR}$ premium) is result similar (Figure 5). All process inks are well cured at printing speeds up to 5000 sheets/hour. Yellow ink is well cured to 6000 sheets/hour and magenta with black inks to 7000 sheets/hour (setting of curing unit $100 \%$ ).

Inks from Siegwerk (Sicura Litho Nutriplast) were all well cured to 7000 sheets/hour (with setting of curing units $100 \%$ ). When the setting of curing units was lower (at printing speed 7000 sheets/hour), the DC decrease and inks cannot be well cured (Figure 6).

Figure 7 shown DC of inks SunCure ${ }^{\circledR}$ FLM from Sun Chemical. All process inks are well cured to 8000 sheets/hour. Cyan and magenta till 10000 and black till 9000 sheets/hour. Second type of inks from Sun Chemical (Suncure ${ }^{\circledR}$ Starluxe) are well cured till 7000 sheets/hour (Figure 8). Problematic is yellow ink (for both sets of Sun Chemical inks), where the decrease in DC is most significant).

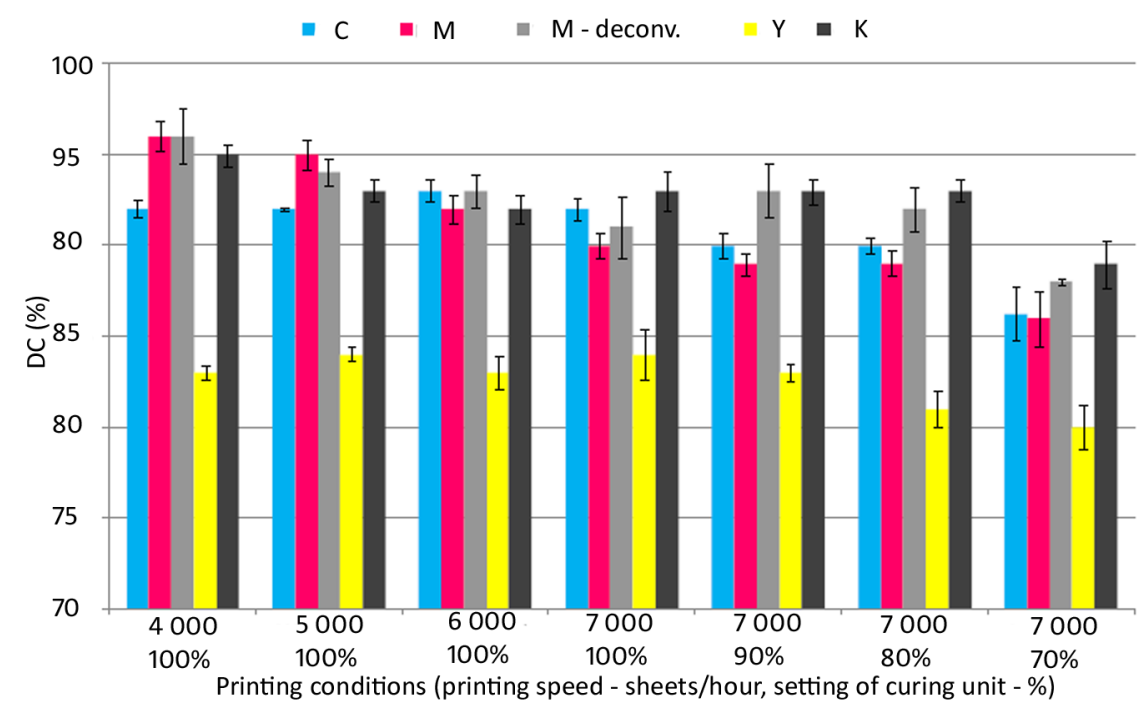

Figure 4: DC of inks UItraCURA ${ }^{\circledR}$ Sens (Flint Group) with different printing conditions

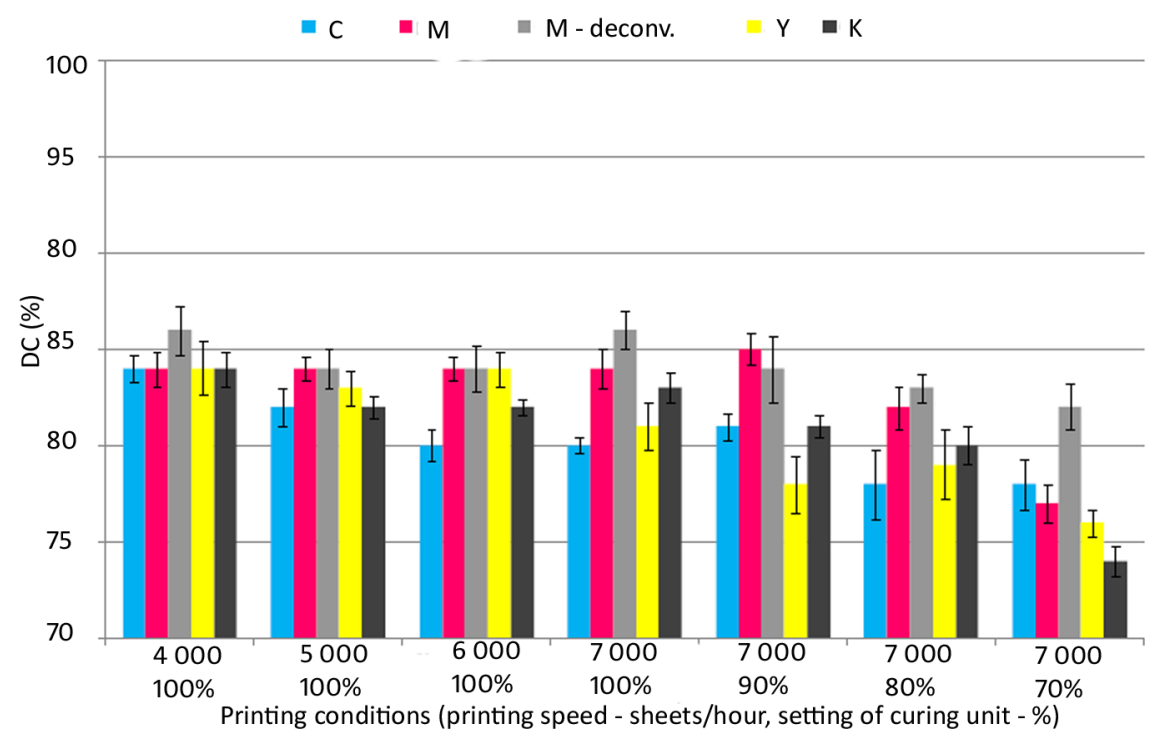

Figure 5: DC of inks NewV pack MGA ${ }^{\circledR}$ premium (Huber Group) with different printing conditions 


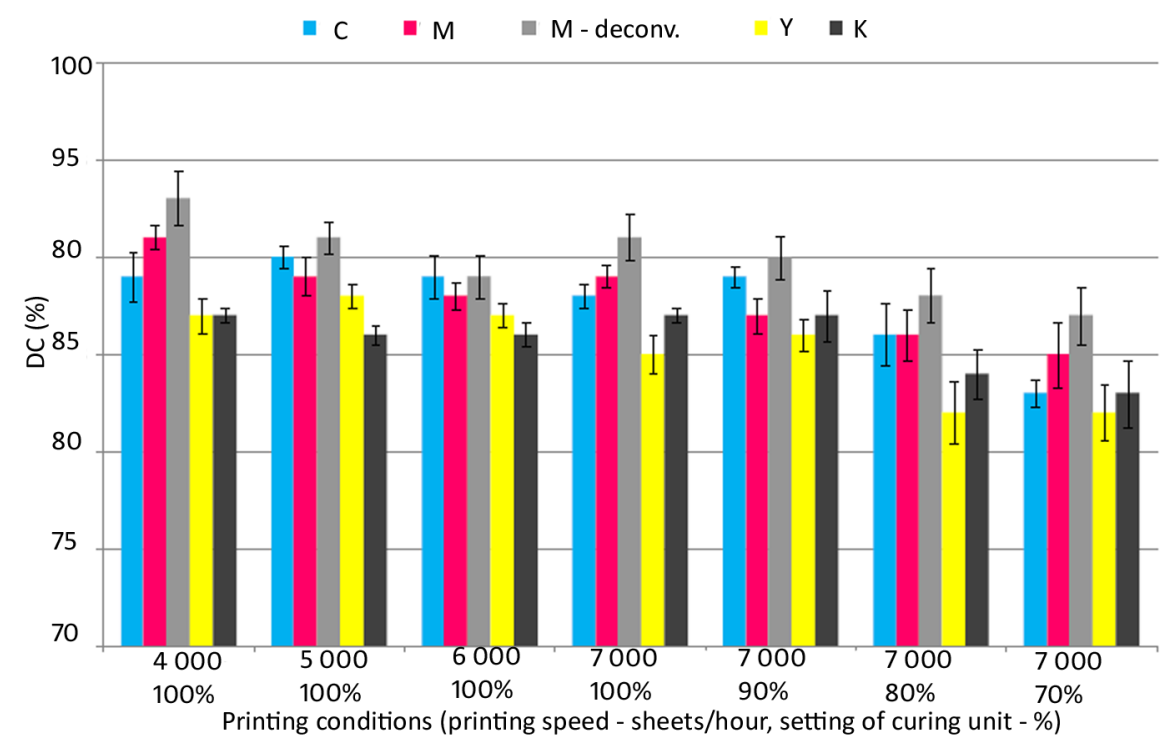

Figure 6: DC of inks Sicura Litho Nutriplast (Siegwerk) with different printing conditions

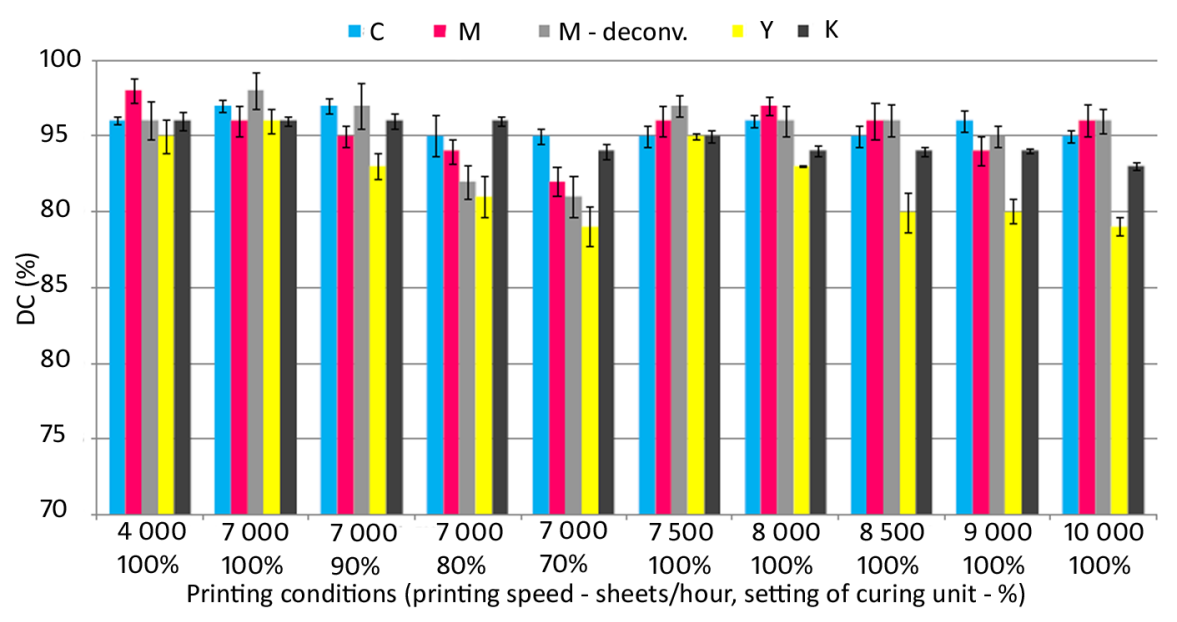

Figure 7: DC of inks SunCure ${ }^{\circledast}$ FLM (Sun Chemical) with different printing conditions

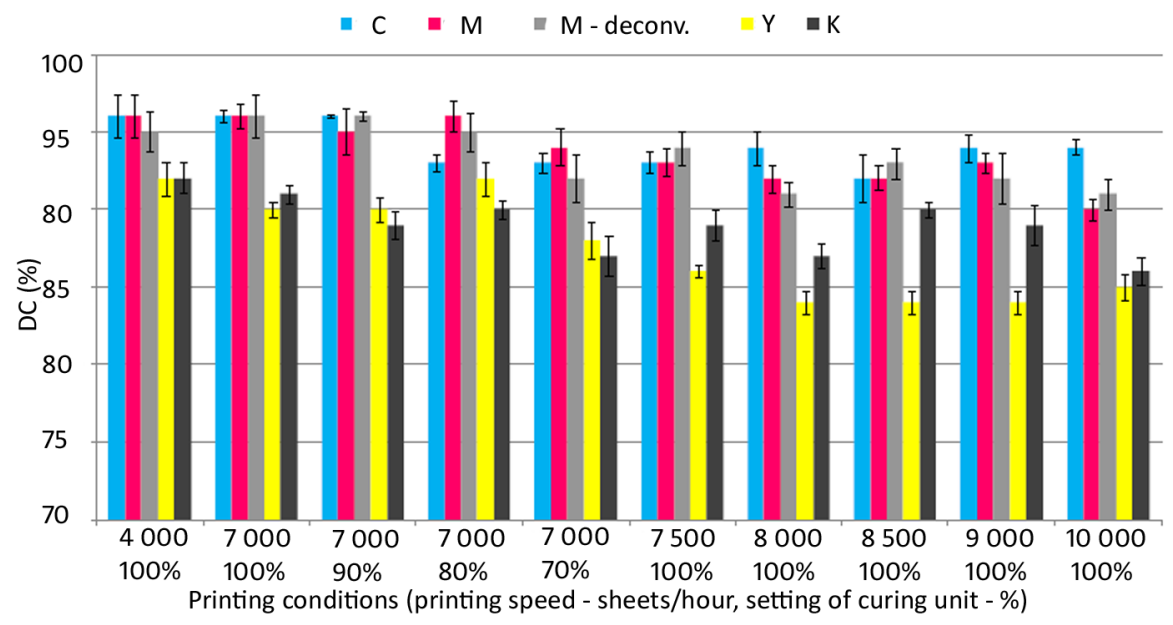

Figure 8: DC of inks Suncure ${ }^{\circledR}$ Starluxe (Sun Chemical) with different printing conditions 
CON-TROL-CURE ${ }^{\circledR}$ UV Fastcheck ${ }^{\mathrm{TM}}$ Strips Key Chart (Figure 9) was used for evaluation of UV dose at different printing speeds. Control strips were stick on printing substrate and with same conditions exposed to UV radiation in printing machine. From colour changes was evaluated UV dose (Table 2).

Table 2: UV doses for different printing speeds evaluated with CON-TROL-CURE ${ }^{\circledR}$ UV Fastcheck ${ }^{\text {TM }}$ Strips Key Chart

\begin{tabular}{|c|c|}
\hline Printing speed (sheets/hour) & UV dose $\left(\mathrm{mJ} / \mathrm{cm}^{2}\right)$ \\
\hline $4000-5000$ & 150 \\
\hline $6000-7000$ & 100 \\
\hline $8000-10000$ & 75 \\
\hline
\end{tabular}

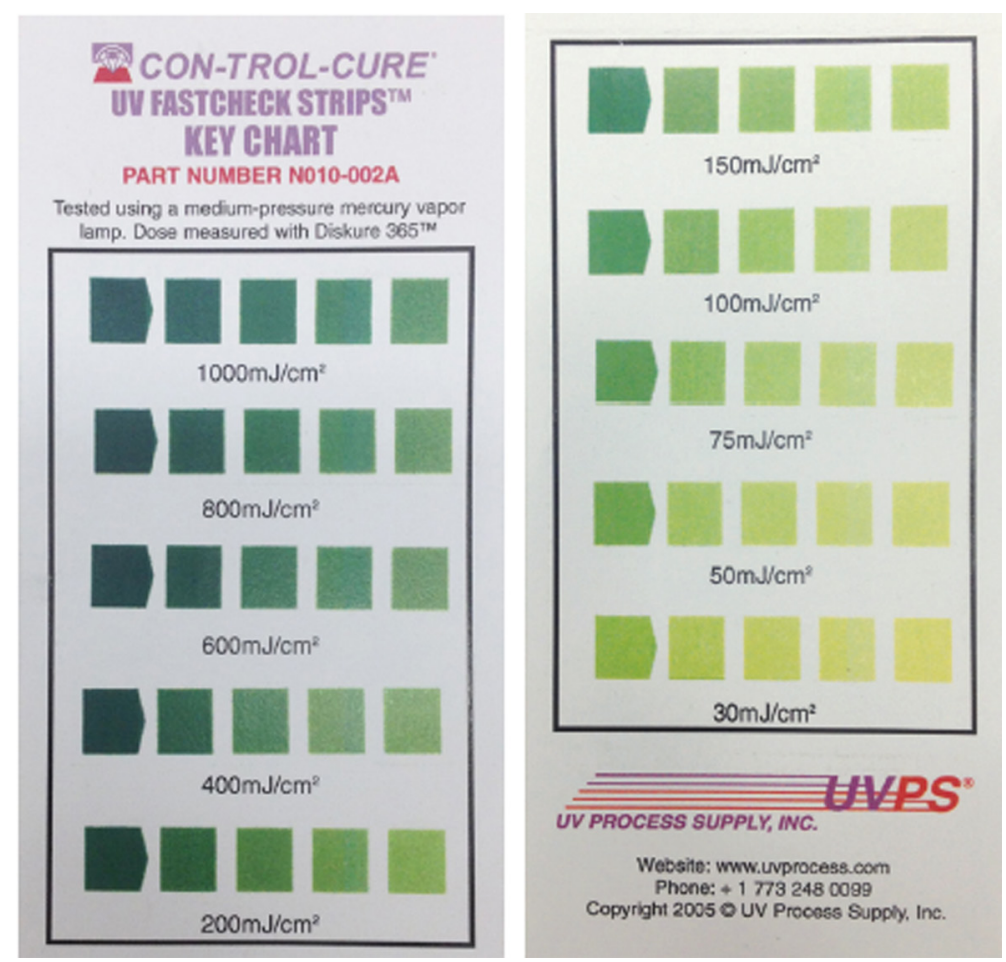

Figure 9: CON-TROL-CURE ${ }^{\circledR}$ UV Fastcheck ${ }^{\text {TM }}$ Strips Key Chart (UV Process Supply, Inc.) for evaluation of UV dose

\section{CONCLUSION}

Important parameter that has to be controlled during print with UV curable inks is their adequate curing. When the curing of UV inks is insufficient, it can result for example in lower ink adhesion, blocking and increased migration of ink components from the cured film. Simple methods used in printing companies to evaluate ink curing (for example Tape test) cannot provide true information. In this work was curing of UV inks and varnish evaluated by FTIR spectroscopy. Results show differences in curing of inks from various producers (Flint Group, Huber group, Siegwerk and Sun Chemical), where all process inks (CMYK) were well cured in range of printing speeds from 5000 to 8000 sheets/hour. The best result was reached with inks from Sun Chemical (SunCure ${ }^{\circledR}$ FLM).

\section{REFERENCES}

[1] Colthup, N. B., Daly, L. H., Wiberley, S. E.: "Introduction to Infrared and Raman Spectroscopy", $3^{\text {rd }}$ ed, (Academic Press, Massachusetts, 1990.).

[2] EuPIA: "Guideline on Printing Inks applied to the non-food contact surface of food packaging materials and articles", Eupia,

URL: http://www.eupia.org/uploads/tx_edm/111114_EuPIA_Guideline_for_Food_Packaging_Inks__November_2011_corr_July_2012_under_review.pdf (last request: 2018-09-18).

[3] Grabitz, A.: "Printing ink legal assessment after the decline of German printing ink ordinance", RadTech Europe Conference 2017, (Rad Tech, Prague, Czech Republic, 2017 ), 17-19. 
[4] Nestlé, Nestlé Guidance Note on Packaging Inks, Argus-analysen, URL: http://www.argus-analysen.de/assets/plugindata/poola/nestle-guidance-note-on-packaginginks-2016-08.pdf (last request: 2018-09-18).

[5] Škola, O., Jašúrek, B., Veselý, D., Němec, P.: "Mechanical properties of polymer layers fabricated via hybrid free radical-cationic polymerization of acrylate, epoxide, and oxetane binders", Progress in Organic Coatings, 101, 279-287, 2016. doi: 10.1016/J.PORGCOAT.2016.08.020

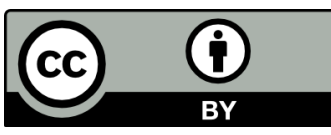

(C) 2018 Authors. Published by the University of Novi Sad, Faculty of Technical Sciences, Department of Graphic Engineering and Design. This article is an open access article distributed under the terms and conditions of the Creative Commons Attribution license 3.0 Serbia (http://creativecommons.org/licenses/by/3.0/rs/). 